\title{
Experimental investigation and multi-objective optimization of micro-wire electrical discharge machining of a titanium alloy using Jaya algorithm
}

\author{
Singh, M. ${ }^{a}$, Ramkumar, J. ${ }^{a}$, Rao, R.V. ${ }^{\mathrm{b},{ }^{*}}$, Balic, J. ${ }^{\mathrm{c}}$ \\ ${ }^{a}$ Department of Mechanical Engineering, Indian Institute of Technology Kanpur, Kanpur, India \\ ${ }^{b}$ Department of Mechanical Engineering, Sardar Vallabhbhai National Institute of Technology Surat, Surat, India \\ ${ }^{\mathrm{C}}$ Faculty of Mechanical Engineering, Laboratory for Intelligent Manufacturing Systems, University of Maribor, Maribor, \\ Slovenia
}

\begin{abstract}
A B S T R A C T
Micro-wire electrical discharge machining (Micro-WEDM) process exhibits superior precision and greater relative accuracy for the efficient machining of difficult-to-machine materials. The micro-slit cutting operation using WEDM process has been experimentally investigated for the objective of analysing the average kerf-loss and responses pertaining to the economic viability of the process viz. average cutting rate and volumetric material removal rate $\left(M R R_{v}\right)$. The experiments are performed using a Tungsten wire of diameter 70 $\mu \mathrm{m}$ on titanium grade 5 alloy (Ti-6Al-4V). Three different controllable process variables (input parameters) associated with the Resistance-Capacitance (RC) based power generator namely discharge energy, wire feed-rate and wire travelling speed are varied to demonstrate their impacts on typical responses such as average kerf-loss, average cutting rate and $M R R_{v}$. The experimental analysis revealed a close relationship that cutting rate bears with discharge energy, wire feed-rate and efficient flushing of molten liquid as well as fine debris particles. An advanced multi-objective optimization technique popularly known as Multi Objective-Jaya (MO-Jaya) algorithm has been adopted for the simultaneous optimization of average kerf-loss, average cutting rate and volumetric material removal rate. The best set of input parameters have been selected to suggest the most optimum responses for micro wire-cutting operations.
\end{abstract}

\section{ARTICLE INFO}

Keywords:

Micro-wire electrical discharge machining (Micro-WEDM); Multi-objective optimization; Titanium alloy; Kerf-loss;

Cutting rate;

Volumetric material removal rate; Feed-rate;

Jaya algorithm;

Multi objective-Jaya algorithm

(MO-Jaya)

*Corresponding author: ravipudirao@gmail.com (Rao, R.V.)

Article history:

Received 2 November 2018

Revised 5 May 2019

Accepted 27 May 2019

(C) 2019 CPE, University of Maribor. All rights reserved.

\section{References}

[1] Di, S., Chu, X., Wei, D., Wang, Z., Chi, G., Liu, Y. (2009). Analysis of kerf width in micro-WEDM, International Journal of Machine Tools and Manufacture, Vol. 49, No. 10, 788-792, doi: 10.1016/j.ijmachtools.2009.04.006.

[2] Narasimhan, J., Yu, Z., Rajurkar, K.P. (2005). Tool wear compensation and path generation in micro and macro EDM, Journal of Manufacturing Processes, Vol. 7, No. 1, 75-82, doi: 10.1016/S1526-6125(05)70084-0.

[3] D’Urso, G., Ravasio, C. (2017). Material-technology index to evaluate micro-EDM drilling process, Journal of Manufacturing Processes, Vol. 26, 13-21, doi: 10.1016/j.jmapro.2017.01.003.

[4] Joshi, S.N., Pande, S.S. (2010). Thermo-physical modeling of die-sinking EDM process, Journal of Manufacturing Processes, Vol. 12, No. 1, 45-56, doi: 10.1016/j.jmapro.2010.02.001.

[5] Tosun, N., Cogun, C., Tosun, G. (2004). A study on kerf and material removal rate in wire electrical discharge machining based on Taguchi method, Journal of Materials Processing Technology, Vol. 152, No. 3, 316-322, doi: 10.1016/j.jmatprotec.2004.04.373. 
[6] Scott, D., Boyina, S., Rajurkar, K.P. (1991). Analysis and optimization of parameter combinations in wire electrical discharge machining, International Journal of Production Research, Vol. 29, No. 11, 2189-2207, doi: $\underline{10.1080 / 00207549108948078 .}$.

[7] Okada, A., Uno, Y., Nakazawa, M., Yamauchi, T. (2010). Evaluations of spark distribution and wire vibration in wire EDM by high-speed observation, CIRP Annals, Vol. 59, No. 1, 231-234, doi: 10.1016/i.cirp.2010.03.073.

[8] Masuzawa, T., Tönshoff, H.K. (1997). Three-dimensional micromachining by machine tools, CIRP Annals, Vol. 46, No. 2, 621-628, doi: 10.1016/S0007-8506(07)60882-8.

[9] Karthikeyan, G., Ramkumar, J., Dhamodaran, S., Aravindan, S. (2010). Micro electric discharge milling process performance: An experimental investigation, International Journal of Machine Tools and Manufacture, Vol. 50, No. 8, 718-727, doi: 10.1016/j.ijmachtools.2010.04.007.

[10] Jahan, M.P., Wong, Y.S., Rahman, M. (2009). A study on the quality micro-hole machining of tungsten carbide by micro-EDM process using transistor and RC-type pulse generator, Journal of Materials Processing Technology, Vol. 209, No. 4, 1706-1716, doi: 10.1016/i.jmatprotec.2008.04.029.

[11] Konak, A., Coit, D.W., Smith, A.E. (2006). Multi-objective optimization using genetic algorithms: A tutorial, Reliability Engineering \& System Safety, Vol. 91, No. 9, 992-1007, doi: 10.1016/i.ress.2005.11.018.

[12] Rao, R.V., Rai, D.P., Balic, J. (2017). A multi-objective algorithm for optimization of modern machining processes, Engineering Applications of Artificial Intelligence, Vol. 61, 103-125, doi: 10.1016/i.engappai.2017.03.001.

[13] Rao, R.V. (2019). Jaya: An advanced optimization algorithm and its engineering applications, Springer International Publishing AG, Cham, Switzerland, doi: 10.1007/978-3-319-78922-4.

[14] Rao, R.V., Rai, D.P., Ramkumar, J., Balic, J. (2016). A new multi-objective Jaya algorithm for optimization of modern machining processes, Advances in Production Engineering \& Management, Vol. 11, No. 4, 271-286, doi: 10.14743/apem2016.4.226.

[15] Rao, R.V. (2016). Jaya: A simple and new optimization algorithm for solving constrained and unconstrained optimization problems, International Journal of Industrial Engineering Computations, Vol. 7, 19-34, doi: 10.5267/j.ijiec.2015.8.004. 


\title{
Eksperimentalna raziskava in večkriterijska optimizacija obdelave $z$ mikrožično erozijo titanove zlitine
} $z$ algoritmom Jaya

\author{
Singh, M. ${ }^{a}$, Ramkumar, J. ${ }^{a}$, Rao, R.V. ${ }^{\text {b, }}{ }^{,}$, Balic, J. ${ }^{\mathrm{c}}$ \\ ${ }^{a}$ Department of Mechanical Engineering, Indian Institute of Technology Kanpur, Kanpur, India \\ ${ }^{b}$ Department of Mechanical Engineering, Sardar Vallabhbhai National Institute of Technology Surat, Surat, India \\ ${ }^{\mathrm{C}}$ Faculty of Mechanical Engineering, Laboratory for Intelligent Manufacturing Systems, University of Maribor, Maribor, Slo- \\ venia
}

\begin{abstract}
POVZETEK
Proces obdelave z mikrožično erozijo (Micro-WEDM) ima vrhunsko natančnost in večjo relativno natančnost za učinkovito obdelavo materialov, ki se težko obdelujejo. Postopek rezanja mikroreže z WEDM je bil eksperimentalno raziskan, da bi analizirali povprečno izgubo v vplivni coni reza in odzive, ki se nanašajo na ekonomsko upravičenost postopka kot sta povprečna hitrost rezanja in stopnja odstranjevanja materiala $\left(M R R_{v}\right)$. Poskusi so izvedeni $\mathrm{z}$ volframovo žico s premerom $70 \mu \mathrm{m}$ na zlitini titana razreda 5 (Ti-6Al-4V). Variirane so tri različne procesne spremenljivke (vhodni parametri), povezane $\mathrm{z}$ generatorjem moči na osnovi upornosti in kapacitivnosti (RC), in sicer razelektritvena energija, podajalna hitrost žice in pomična hitrost žice, da raziščemo njihov vpliv na značilne odzive, kot so povprečna izguba v vplivni coni reza, povprečna hitrost rezanja in $M R R_{v}$. Eksperimentalna analiza je pokazala tesno povezanost hitrosti rezanja z razelektritveno energijo, podajalno hitrostjo žice in učinkovitim izpiranjem staljene tekočine ter drobnih delcev. Za sočasno optimizacijo povprečne izgube v vplivni coni reza, povprečne hitrosti rezanja in hitrosti odstranjevanja materiala je bil uporabljen napredni pristop večkriterijske optimizacije, imenovan tudi večkriterijski-Jaya (MOJaya) algoritem. Izbrana je bila najboljša kombinacija vhodnih parametrov, ki omogoča optimalne rezultate pri obdelavi z mikrožično erozijo.
\end{abstract}

(c) 2019 CPE, University of Maribor. All rights reserved.

\section{PODATKI O ČLANKU}

Ključne besede:

Obdelava z mikrožično erozijo

(Micro-WEDM);

Večkriterijska optimizacija;

Titanove zlitine;

Izguba v vplivni coni reza;

Stopnja rezanja;

Stopnja volumetričnega odstranjevanja materiala;

Podajalna hitrost;

Algoritem Jaya;

Večkriterijski algoritem Jaya

(MO-Jaya)

*Kontaktna oseba:

ravipudirao@gmail.com

(Rao, R.V.)

Zgodovina članka:

Prejet 2. novembra 2018

Popravljen 5. maja 2019

Sprejet 27. maja 2019 